\title{
Effect of Circuit Structure on Odor Representation in the Insect Olfactory System
}

\author{
Adithya Rajagopalan ${ }^{1}$ and Collins Assisi ${ }^{2}$
}

https://doi.org/10.1523/ENEURO.0130-19.2020

${ }^{1}$ Janelia Research Campus, Howard Hughes Medical Institute, Ashburn, Virginia 20147 and ${ }^{2}$ Division of Biology, Indian Institute of Science Education and Research, Pune 411008, India

\begin{abstract}
In neuroscience, the structure of a circuit has often been used to intuit function-an inversion of Louis Kahn's famous dictum, "Form follows function" (Kristan and Katz, 2006). However, different brain networks may use different network architectures to solve the same problem. The olfactory circuits of two insects, the locust, Schistocerca americana, and the fruit fly, Drosophila melanogaster, serve the same function-to identify and discriminate odors. The neural circuitry that achieves this shows marked structural differences. Projection neurons (PNs) in the antennal lobe innervate Kenyon cells (KCs) of the mushroom body. In locust, each KC receives inputs from $\sim 50 \%$ of PNs, a scheme that maximizes the difference between inputs to any two of $\sim 50,000 \mathrm{KCs}$. In contrast, in Drosophila, this number is only $5 \%$ and appears suboptimal. Using a computational model of the olfactory system, we show that the activity of KCs is sufficiently high-dimensional that it can separate similar odors regardless of the divergence of PN-KC connections. However, when temporal patterning encodes odor attributes, dense connectivity outperforms sparse connections. Increased separability comes at the cost of reliability. The disadvantage of sparse connectivity can be mitigated by incorporating other aspects of circuit architecture seen in Drosophila. Our simulations predict that Drosophila and locust circuits lie at different ends of a continuum where the Drosophila gives up on the ability to resolve similar odors to generalize across varying environments, while the locust separates odor representations but risks misclassifying noisy variants of the same odor.
\end{abstract}

Key words: Drosophila; locust; mushroom body; olfaction; optimality; sparseness

\section{Significance Statement}

How does the structure of a network affect its function? We address this question in the context of two olfactory systems that serve the same function to distinguish the attributes of different odorants, but do so using markedly distinct architectures. In the locust, the probability of connections between projection neurons and Kenyon cells-a layer downstream-is nearly $50 \%$. In contrast, this number is merely $5 \%$ in Drosophila. We developed computational models of these networks to understand the relative advantages of each connectivity. Our analysis reveals that the two systems exist along a continuum of possibilities that balance two conflicting goals-separating the representations of similar odors while grouping together noisy variants of the same odor.

\section{Introduction}

Neural circuits encode a variety of stimuli and perform a wide range of computations. The structure of the neural circuit (i.e., the organization and statistics of the connectivity

Received April 4, 2019; accepted February 23, 2020; First published April 28, 2020.

The authors declare no competing financial interests. between neurons in the circuit) plays a key role in restricting the kinds of computations that the circuit can perform (Marr, 1969; Albus, 1971; Hopfield and Tank, 1986). Understanding what different structural organizations imply for circuit

Author contributions: A.R. and C.A. designed research; A.R. and C.A. performed research; A.R. and C.A. contributed unpublished reagents/analytic tools; A.R. and C.A. analyzed data; A.R. and C.A. wrote the paper. 
function is an integral step toward generating a complete picture of brain function. These structure-function relationships are of particular interest in circuits that are trying to accomplish the same overarching goal while making use of different structural parameters. What advantages do the different parameter regimes provide in such situations? One such instance that has been explored recently (Jortner et al., 2007; Jortner, 2013; Litwin-Kumar et al., 2017) is the functional effect of different densities of connections across species in the antennal lobe-mushroom body (MB) circuit of the insect olfactory system.

The insect olfactory system is arguably one of the most well characterized neural circuits. Its compactness and simplicity, combined with the powerful genetic tools available, have allowed a detailed understanding of its structure and function. The circuit begins at the olfactory sensory neurons that convert odorant information from the environment into electrical signals that are passed on to higher brain regions (Hallem and Carlson, 2004, 2006; Fisek, 2014). The second level of the circuit is the antennal lobe $(A L)$, where the principal excitatory neurons-projection neurons (PNs)-represent odors as dense spatiotemporal firing patterns (Laurent et al., 1996; Wehr and Laurent, 1996; Wilson and Laurent, 2005). The $A L$ then feeds information to the $M B$, where Kenyon cells (KCs) represent the odor as a spatially and temporally sparse pattern of firing (Perez-Orive et al., 2002; Turner et al., 2008). A high spiking threshold and inhibitory inputs to KCs from a pair of large GABAergic neurons (Papadopoulou et al., 2011; Lin et al., 2014; Masuda-Nakagawa et al., 2014) maintain the sparseness of $\mathrm{KC}$ responses. The inhibitory GABAergic neurons are graded neurons whose membrane voltage is mediated by the activity of the $\mathrm{KCs}$, thus forming a feedback inhibition loop (Fig. 1). Synapses immediately downstream of the KCs are plastic and thought to be the primary locus of associative memory in the insect (Heisenberg, 2003; Hige et al., 2015a). KCs converge on to the MB output neurons (MBONs). From the MBONs onward, neuronal activity is related more to behavioral output than to stimulus representation (Aso et al., 2014; Hige et al., 2015a).

While the overarching goal of the MB circuit-to distinctly represent odors so as to facilitate learning and appropriate behavioral responses-appears to be conserved across species, the number of connections received from the $\mathrm{AL}$ to a given $\mathrm{KC}$ varies significantly. In the fruit fly, a sparse $\sim 5 \%$ of all PNs synapse onto each $\mathrm{KC}$, whereas in the locust, this number is dense ( $\sim 50 \%$; Fig. 1; Jortner et al., 2007; Caron et al., 2013). The 50\% connectivity seen

C.A. was funded by the DBT-Wellcome Trust India Alliance through Intermediate Fellowship IA/I/11/2500290 and by the Indian Institute of Science Education and Research Pune. A.R. was funded by an INSPIRE Fellowship, Department of Science and Technology, India. We thank members of the Nadkarni and Assisi laboratories and Dr. Aurnab Ghose for useful discussions.

Correspondence should be addressed to Adithya Rajagopalan at rajagopalana@janelia.hhmi.org; or Collins Assisi at collins@iiserpune.ac.in.

https://doi.org/10.1523/ENEURO.0130-19.2020

Copyright @ 2020 Rajagopalan and Assisi

This is an open-access article distributed under the terms of the Creative Commons Attribution 4.0 International license, which permits unrestricted use, distribution and reproduction in any medium provided that the original work is properly attributed. in the locust olfactory system is thought to maximize the differences between the inputs received by individual KCs (Jortner et al., 2007; Jortner, 2013). The 5\% connectivity observed in Drosophila must then make it a suboptimal classifier. The combinatorial arguments that have been posited thus far do not consider the full spatiotemporal extent of an odor-evoked pattern of activity in the antennal lobe. To understand the implications of these contrasting connectivities, we tested the response of the fly and the locust olfactory networks to two different kinds of inputs: one, where odors were represented as spatiotemporal patterns of activity by $\mathrm{AL}$ neurons, and another, where odors were represented only by the identity of active PNs. We show that an identity code allows a broad range of connection densities, including those seen in both the fly and locust, to distinguish different odors. However, with temporal variations, denser connectivities between PNs and KCs maximize the distance between odor representations. The sensitivity of the locust olfactory system, due to its dense connectivity, comes at a cost. Under changing environmental conditions, the same odor may generate different representations in PN space that the locust could potentially misclassify as distinct odors. Such misclassifications are less likely in the Drosophila circuit where $\mathrm{PN}-\mathrm{KC}$ connections are sparse. To elucidate the logic behind these connectivities, we simulated the distinct architectures of each insect. In Drosophila, all the sensory neurons expressing a particular receptor type synapse onto PNs in a spatially circumscribed structure called a glomerulus. Sister PNs, which receive inputs from olfactory receptor neurons (ORNs) at a particular glomerulus, tend to fire in a highly correlated manner (Kazama and Wilson, 2009), though this is not the case in related mammalian cells (Dhawale et al., 2010), where the activity, though correlated, is different. In contrast, locust glomeruli receive input from multiple ORN types. We show that the glomerular architecture of the fruit fly improves the ability of the network to distinguish odors despite a low probability of $\mathrm{PN}-\mathrm{KC}$ connections. Our simulations predict that the fruit fly and locust circuits lie at different ends of a continuum where the fruit fly gives up on resolution in odor space so that it can generalize across varying environments. This implies that very similar odors may be misclassified as the same odor as they are too similar to be resolved. The locust, on the other hand, maximally separates odor representations but runs the risk of misclassifying the same odor under different conditions.

\section{Materials and Methods}

Temporally patterned odor representations in $A L$ circuits. We modeled the odor representation in the $A L$ in two ways. First, as a static representation consisting of a binary vector of length 900 (number of model PNs). Each element of the vector indicated only whether a particular $P N$ was active (if the value at that position was 1$)$ or not ( 0 ; Fig. 2a). The second representation incorporated the temporal evolution of the odor. In the locust AL, odors elicit a temporal pattern of activity in PNs that begins with the onset of the odor. In experimental recordings, not all PNs 


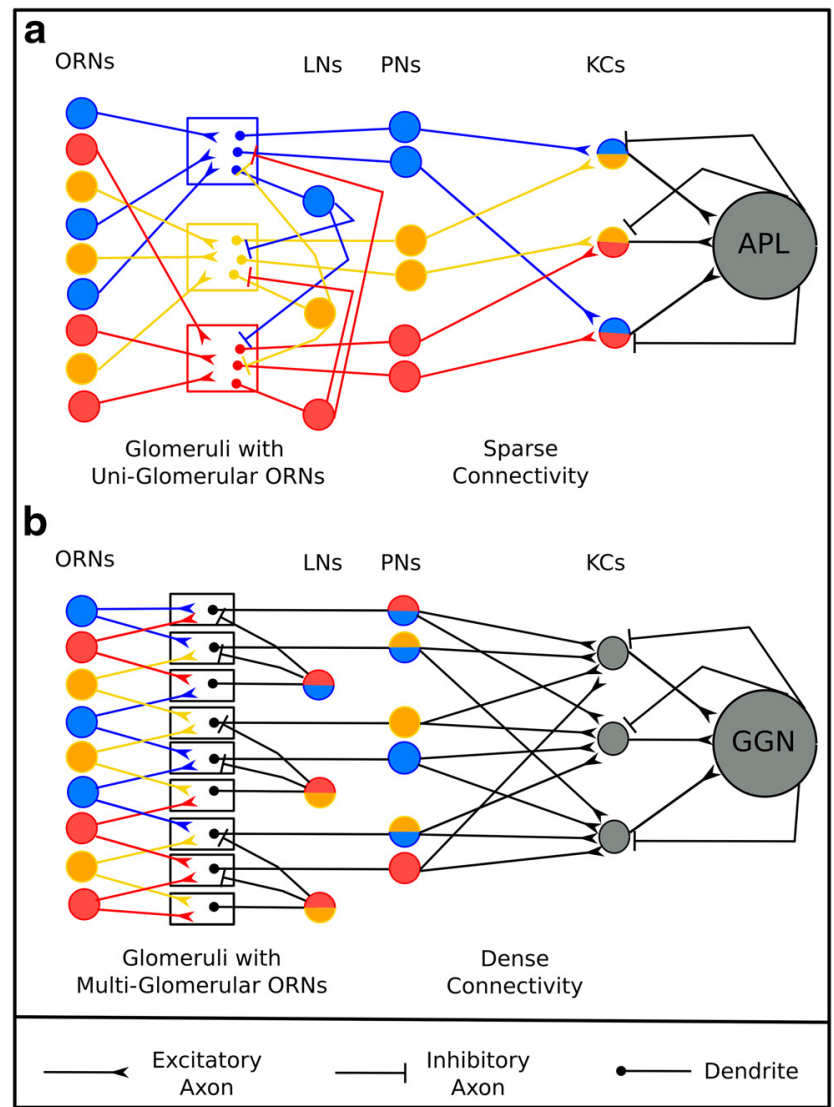

Figure 1. A schematic of the insect olfactory system. $\boldsymbol{a}, \boldsymbol{b}, \mathrm{A}$ schematic of the olfactory system contrasting the structural parameters of the circuit in Drosophila melanogaster (a) and Schistocerca americana (b).

show an odor-specific response that begins immediately on odor onset. Several neurons show increased activity many milliseconds after odor onset. Some PNs can show complex responses such as an increased level of activity to both odor onset and offset. However, it is likely that the onset and offset responses are largely seen in nonoverlapping groups of PNs (Saha et al., 2017). Here, we simulated PN spiking activity as continuous bursts. The spatiotemporal pattern generated by the PN population was defined by the onset, offset, and duration of PN bursts. Another important aspect to consider was the presence of oscillations in the local field potential (LFP) in the 20-30 Hz frequency range (Laurent, 1996) in the AL of locusts. Similar oscillations have also been observed in intracellular recordings from Drosophila AL (Tanaka et al., 2009). The presence of such oscillations suggests that odor-induced PN responses are correlated with more PNs spiking at the peak of the LFP than at other phases. The oscillations also provide a natural time scale to partition the PN response into smaller 50 ms epochs (the duration of one cycle at $20 \mathrm{~Hz}$ ). We measured the time to odor initiation and the duration of a continuous $\mathrm{PN}$ response in units of epochs. The statistics of the number and timing of PN spikes were extracted from a survey of the literature (Table 1; Laurent et al., 1996; Wehr and Laurent, 1996;
Stopfer et al., 2003; Wilson and Laurent, 2005). We adapted these results to design a matrix representation of PN activity. This consisted of a $900 \times 3000$ matrix of 1 and $0 \mathrm{~s}$ (Fig. 3). Each row represented 1 of 900 PNs, and each column of the matrix represented the activity of all PNs over a $1 \mathrm{~ms}$ time interval. The parameters (and their values) used in this process (to simulate $1 \mathrm{~s}$ of odor delivery and a $3 \mathrm{~s}$ response) are listed below (note that all variables are normally distributed, and values represent the mean \pm SD unless mentioned otherwise).

To generate a population PN response, a value used to specify the percentage of active neurons was drawn from a normal distribution, with mean and variance given in Table 1. This value was used as a probability threshold to decide whether a given $\mathrm{PN}$ fires or not. For each of the 900 PNs, a uniform random number was drawn to decide whether that PN was activated by the odor. If the random value was less than the probability threshold chosen, then the neuron was activated by the odor. A value of the basal firing rate (per second) was drawn from a normal distribution with the appropriate mean and SD (Table 1), and spikes equaling three times the value drawn were uniformly and randomly distributed over the 3000 time points. A value for odor-induced firing rate was drawn from a normal distribution, as were the number of active epochs and the number of epochs before odor-induced activity. These three values provide information about which of the LFP oscillation cycles additional spikes needed to be added to the activity of the particular neuron, as well as how many spikes were to be added in a single epoch. These spikes were then distributed in each of the "active" epochs in such a way that the spike was more likely to occur at the center of the epoch (corresponding to the peak of the LFP) than at the ends. If the neuron was not odor activated, then it fired at its basal firing rate, as described earlier.

These attributes were calculated for each of the 900 PNs to generate a complete spatiotemporal pattern describing an odor. An odor was defined by the specific PNs that were activated and the parameters drawn from the distributions quantified in Table 1. In different trials of the same odor, the PNs that were activated, as well as their parameters, remained the same. However, the exact timing of the spikes in the active epochs changed.

The timing of spikes was drawn randomly (within specified "active" epochs) for each trial. In contrast, two odors differ not only in the timing of spikes of active PNs but also in the identity of the active PNs.

Whether a PN was active or not was independent of whether other PNs were active. This reflected the multiglomerular organization seen in locust. To mimic a fly-like glomerular organization where sister PNs fire in a correlated manner, PNs were divided in 50 groups of six (note that here we simulated $300 \mathrm{PNs}$ and not 900 , which is in agreement with the number seen in the fly). The grouping reflected the glomerular architecture in Drosophila. Five of these 50 groups were chosen to contain active neurons. The other four parameters mentioned in Table 1 were then 
a
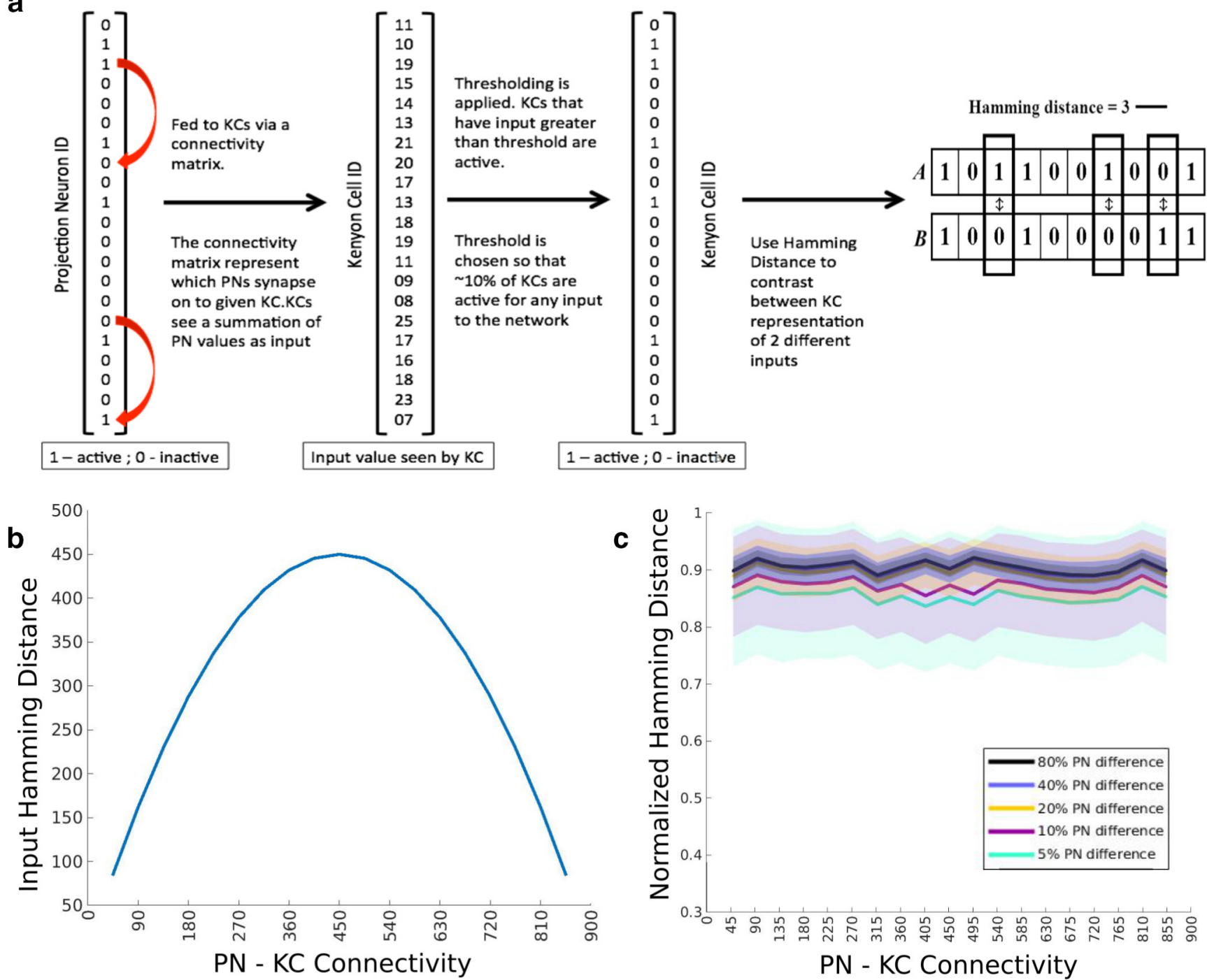

Figure 2. The $50 \%$ connectivity does not maximally separate $\mathrm{KC}$ representations when PN inputs are static. a, The threshold model of $\mathrm{KCs}$. The left-most vector represents the PN activity. This is combined through a connectivity matrix to give the input seen by each KC (a 50,000 -element-long vector). Thresholding is then applied to define spiking KCs. $\boldsymbol{b}$, The Hamming distance between inputs seen by two $\mathrm{KCs}$ is calculated for all possible pairs and averaged and plotted as a function of the PN-KC connectivity. c, The mean ( \pm SD) normalized Hamming distance between the activity of KC networks driven by two different inputs is plotted on the $y$-axis as a function of the PN-KC connectivity. Different shades plot the distance between odor representations that differed in $5-80 \%$ of the active PNs.

chosen for these active neurons. To simulate a new odor that was distinct from a previously described one, one to five of the active glomeruli in the first odor were changed randomly (Fig. 3, instance of a simulated odor).

Table 1: Statistics of PN spikes

\begin{tabular}{ll}
\hline $\begin{array}{l}\text { Percentage of active } \\
\text { neurons }\end{array}$ & $(0.2 \pm 0.05) \times$ number of PNs \\
$\begin{array}{c}\text { Basal firing rate } \\
\text { Odor induced firing } \\
\text { rate }\end{array}$ & $3.87 \pm 2.23$ spikes/s \\
$19.53 \pm 10.67$ spikes $/ \mathrm{s}$ \\
$\begin{array}{c}\text { Number of active } \\
\text { epochs }\end{array}$ & $8 \pm 4$ cycles of LFP \\
$\begin{array}{c}\text { Number of epochs } \\
\text { before activity }\end{array}$ & $\begin{array}{c}\text { Number of LFP cycles drawn from a } \\
\text { uniform integer distribution ranging from } \\
1 \text { to } 20\end{array}$ \\
\end{tabular}

Neuron and synapse implementation. The spatiotemporal pattern that was generated using specific attributes for PN spike statistics described above was used to stimulate a layer of $50,000 \mathrm{KCs}$. We systematically varied $\mathrm{PN}-\mathrm{KC}$ connections and computed the corresponding $\mathrm{KC}$ responses to several odors. PN-KC synapses are cholinergic (Yasuyama and Salvaterra, 1999) and were modeled as such (Eqs. 1-3; Destexhe et al., 1994; Bazhenov et al., 2001; Perez-Orive et al., 2002; Turner et al., 2008). Each PN spike released a fixed amount of neurotransmitter $\mathrm{T}$. This was used to drive postsynaptic KCs. The synaptic currents were given by the following:

$$
I_{\text {syn }}=g_{\text {syn }} \times[O] \times\left(V-E_{\text {syn }}\right),
$$


a

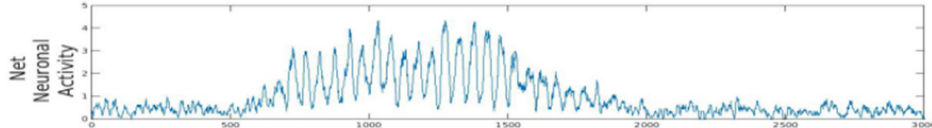

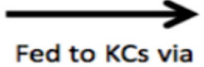
a connectivity matrix. $T$

values are calculated for each $\mathrm{PN}$ and then summed to produce the $T$ values seen by each KC.

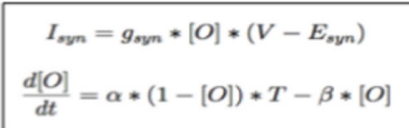

$\mathrm{I}_{\text {syn }}$ is calculated and fed into the calculation of voltage

$C_{m} * \frac{d V}{d t}=-g_{L} *\left(V-E_{L}\right)-I_{s y n}$
Activity of KCs is noted over the entire $3000 \mathrm{~ms}$ window

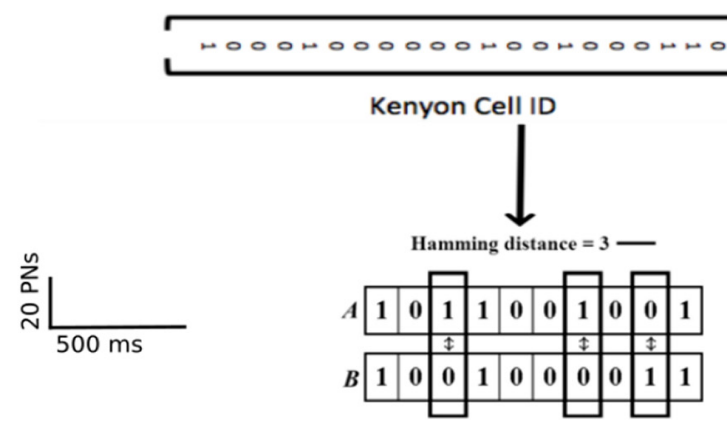

b

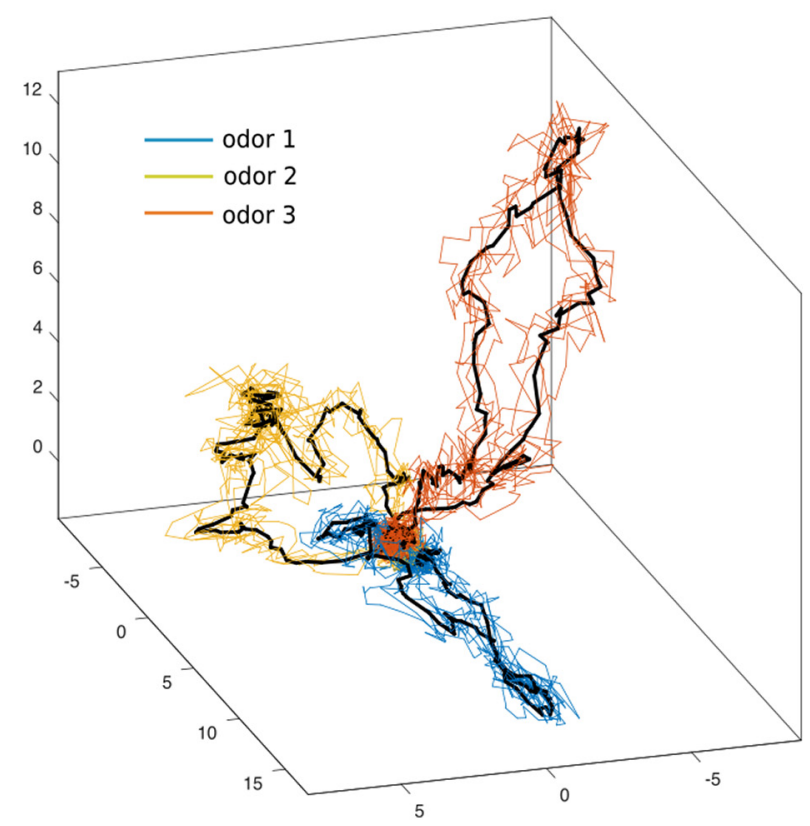

C
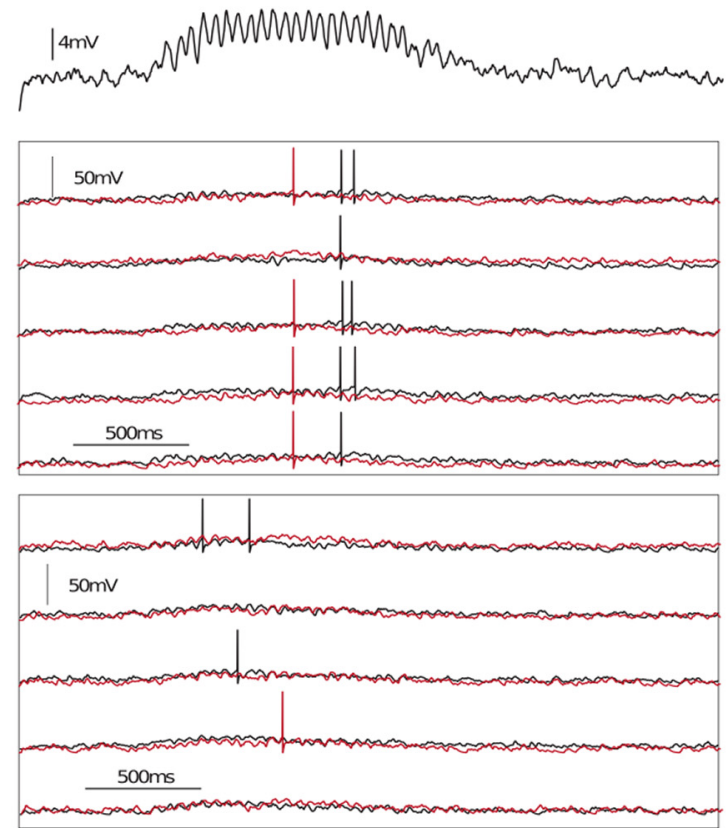

Figure 3. Simulation of temporally patterned PN inputs to a KC network. $\boldsymbol{a}$, The matrix on the left represents the activity of a set of 900 PNs. Each row shows the activity of a single PN during a $3000 \mathrm{~ms}$ time period. Blue dots show the time of a spike. The red region represents the time during which the odor was presented. Top, A summation of the activity of the entire PN network is shown clearly indicating the oscillations in the net PN activity. This input was used to calculate $T$ and $I_{\text {syn }}$ (the synaptic input to KCs). The differences between the population representations of two inputs were calculated using the Hamming distance. $\boldsymbol{b}$, The mean population response of 900 PNs projected onto the first three principal components for three odors is shown by the black traces. Individual trials are shown by the colored traces. $\boldsymbol{c}$, The mean membrane potential of all KCs shows a $20 \mathrm{~Hz}$ oscillation. Bottom, The response of two KCs (in red and black traces) to two different odors. Only the first odor evokes a consistent response from this particular KC across five odor trials (middle). The second odor does not lead to reliable spiking in this example KC. 
where,

$$
\begin{aligned}
& \frac{d[O]}{d t}=\alpha \times(1-[O]) \times T-\beta \times[O] \\
T= & A \times \Theta \times\left(t_{0}+t_{\max }-t\right) \times\left(t-t_{0}\right) .
\end{aligned}
$$

In these equations, the constants were as follows:

$$
\begin{gathered}
\alpha=0.94 \mathrm{~ms}^{-1}, \beta=0.18 \mathrm{~ms}^{-1}, g_{\mathrm{syn}}=0.05 \frac{\mathrm{mS}}{\mathrm{cm}^{2}} \\
E_{\text {syn }}=0 \mathrm{mV} \text { and } t_{\max }=0.3 \mathrm{~ms}
\end{gathered}
$$

where $\Theta$ is the Heaviside function, [O] is the open probability of the ion channels on the $\mathrm{KC}$ membrane, and $T$ represents the amount of neurotransmitter released by a given $\mathrm{PN} . t_{0}$ is the time of the last spike, and $t_{\max }$ is the duration for which the neurotransmitter was released. KCs were modeled as leaky integrate-and-fire neurons (Turner et al., 2008; Papadopoulou et al., 2011), as follows:

$$
C_{m} \frac{d V}{d t}=-g_{L}\left(V-E_{L}\right)-I_{\text {syn }}
$$

Here, $g_{L}=0.089 \frac{\mathrm{mS}}{\mathrm{cm}^{2}}, C_{m}=1 \frac{\mu F}{\mathrm{~cm}^{2}}$, and $E_{L}=-65 \mathrm{mV}$. The $\mathrm{KC}$ generated a spike when $V>V_{\text {thresh. }}$ The membrane potential was reset to $-65 \mathrm{mV}$ at the time point immediately after the spike. We simulated an array of 50,000 such KCs that responded to a 3000-ms-long input from PNs.

Classification and distance metrics. To quantify the difference between the representations of two odors by the same neuronal population, we used the Hamming distance. Elements of the KC activity vector were set to 1 if that $\mathrm{KC}$ fired a spike during the odor presentation, and 0 otherwise.

The Hamming distance calculates the number of bits that differ between the two vectors (Fig. 2, example). In some figures, we used a normalized version of this metric that divides twice the Hamming distance by the total number of active neurons in both vectors being compared. To illustrate this metric, consider a vector representing the activity of 100 neurons. Consider, in one scenario, that 10 of these neurons were active for odor $A$, and a different set of 10 nonoverlapping neurons for odor $B$. The Hamming distance between these odor representations would be 20 . In another scenario, 20 neurons were activated for odor $A$ and 20 nonoverlapping neurons for odor B, the Hamming distance would be 40 . However, in both cases the two odors were maximally different from one another; that is, they did not overlap. In contrast, the normalized Hamming distance for both cases described above would take a maximum value of 1 . The normalized Hamming distance may be thought of as a measure of the degree of overlap between odor representations. If two odors stimulate strictly nonoverlapping $\mathrm{KCs}$, the distance between the representations would be 1 regardless of the number of active KCs. This normalization was also necessary to visualize the distance between odor representations, particularly when the PN-KC connections were dense (>50\%). Dense connectivity regimes showed a large trial-trial variation in the number of active KCs.

In addition to using the normalized Hamming distance to visualize the distance between odor representations, we used two classification algorithms (k-medoids clustering and nonclassical multidimensional scaling) to visualize and classify high-dimensional KC representations of odors. In both of these classification algorithms, we first defined the pairwise Hamming distance between the KC vectors of all simulated odor representations. The algorithm ( $k$-medoids clustering using MATLAB) iteratively minimizes the within-cluster distance while maximizing the distance across clusters. Unlike the k-means clustering algorithm that calculates a center for each cluster as the mean of the cluster, the k-medoids algorithm treats an existing data point as the center of the cluster and measures all within-cluster distances from that point. We also performed a multidimensional scaling analysis using the mdscale function in MATLAB. The algorithm maps points from the high-dimensional KC space to a plane while preserving the pairwise distance relationship between all of the data points.

Data availability. The code/software described in the article is freely available online at http://modeldb.yale.edu/ 261877. The access code for the online repository is 0000. The code is also available in Extended Data 1.

\section{Results}

In the locust, each $\mathrm{KC}$ receives input from nearly half of the antennal lobe PNs. This pattern of connectivity maximizes the difference between inputs to any 2 of the $\sim 50,000$ Kenyon cells in the mushroom body (Fig. $2 b$; Jortner et al., 2007). Given the large number of possible combinations of inputs to KCs, it is highly unlikely that the combination of PNs that synapse onto a given $\mathrm{KC}$ will be exactly the same as that synapsing onto any other $\mathrm{KC}$. In contrast, if the PN-KC connection probability were 5\% (e. g., that seen in Drosophila), the number of total possible PN combinations would be nearly $99 \%$ lower than if the $\mathrm{PN}-\mathrm{KC}$ connection probability were $50 \%$, making it more likely for two KCs to share the same inputs (Fig. 2b; Jortner et al., 2007; Jortner 2013). What advantages do this seemingly suboptimal scheme offer? We addressed this conundrum by simulating a model $\mathrm{KC}$ network that received realistic $P N$ input. Using the distance between $\mathrm{KC}$ odor representations, and the classification accuracy of the network, as a proxy for the ability of the animal to distinguish odors, we determined the circumstances under which different circuit connectivities confer specific advantages in odor discrimination.

\section{A PN identity code allows a wide range of connectivities to distinctly represent odors}

If each $\mathrm{KC}$ sees $m$ of $n$ PNs, then the maximum number of combinations would be obtained for $m=\frac{n}{2}$ (Fig. $2 b$ ). However, it is the response of KCs that is read by subsequent layers, not PN input. The $\mathrm{KC}$ response may be thought of as a nonlinear transformation of the summed input from the PNs. KCs act as coincidence detectors 
that integrate presynaptic input that arrives within short temporal windows on the order of $\sim 50 \mathrm{~ms}$ (Perez-Orive et al., 2002, 2004; Gruntman and Turner, 2013). KCs fire only if a sufficient number of spikes fall within the integration window. Therefore, we first investigated whether the previously hypothesized (Jortner et al., 2007) optimal connection probability from $\mathrm{PNs}$ to $\mathrm{KCs}$ remains optimal despite the threshold imposed by the $\mathrm{KC}$ response and whether a lower connection probability is indeed suboptimal.

We tested this hypothesis using a simple threshold model of KCs and determined how distinctly the KC population output represented different odors. We modeled the input to KCs as a binary vector of length 900 . This captured a single snapshot of the activity of the AL circuit (Jortner, 2013; Litwin-Kumar et al., 2017; Fig. 2a). In the locust $\mathrm{AL}$, the duration of each cycle of the $20 \mathrm{~Hz}$ oscillatory local field potential provides a natural time scale to define the duration of a snapshot. We then calculated the response of $\mathrm{KCs}$ to this input for different values of PN$\mathrm{KC}$ connectivity. We varied the number of projections from $\mathrm{PNs}$ to $\mathrm{KCs}$ such that each $\mathrm{KC}$ received inputs from $5 \%$ to $95 \%$ of all PNs (in steps of $5 \%$ ). We simulated different odors by randomly shuffling the PN activity vector. If the summed activity of all the PNs that were connected to the same $\mathrm{KC}$ exceeded a threshold, we labeled the KC as active and set its response to 1 . Increasing the density of connections from PNs to KCs increased the number of active $\mathrm{KCs}$ for the same input vector. Changes in the sparseness of the KC output vector can lead to a change in the distance between odor representations. Our goal was to calculate the overlap between output vectors, independent of the sparseness of the representation. Therefore, for each connection probability we adjusted the response threshold of KCs such that only $10 \%$ of the $50,000 \mathrm{KCs}$ simulated crossed the threshold. (PerezOrive et al., 2002; Turner et al., 2008). This ensured that changes in the distance between odor representations were solely due to changes in the $\mathrm{PN}-\mathrm{KC}$ connectivity and were not confounded by connectivity-dependent changes in the sparseness of the KC response. We simulated four sets of inputs consisting of 101 PN odor representations. Within each of the four sets of simulated odors, the input vectors differed from each other by varying amounts: $5,10,20,40$, or $80 \%$, respectively. For example, consider the 900 PNs whose activity represented a given odor, "A." Approximately $20 \%$ of these PNs would be active. Another odor, "B," in the input set would differ from $A$ by $10 \%$ if 90 of the 900 PNs changed their activity state from active to inactive or vice versa when compared with $\mathrm{A}$. We then calculated the normalized Hamming distances between odor pairs belonging to each group and compared the distances obtained for different PN-KC connection probabilities. The ability of the $\mathrm{KC}$ population to distinctly represent odors showed no dependence on the connectivity between the two regions (Fig. $2 \mathrm{c}$ ) regardless of the degree of similarity between the $\mathrm{PN}$ representations of odors. This counterintuitive result arises from the fact that even at low connectivity values the number of ways to choose inputs to KCs is more than a hundred orders of magnitude greater than the number of KCs in the network (Litwin-Kumar et al., 2017; see Discussion). Therefore, when odor distances were measured in terms of the output of $\mathrm{KCs}$, both the Drosophila (5\% PN-KC connectivity) and the locust olfactory network (50\% connectivity) were equally capable of distinguishing between similar odors.

\section{Inclusion of PN temporal patterning reveals the functional differences between connectivities}

In response to an odor presentation, $\mathrm{AL}$ neurons generate a dynamic pattern that evolves reliably and over multiple time scales. This spatiotemporal patterning is thought to progressively decorrelate the representations of similar odorants (Wiechert et al., 2010) and make them more easily discriminable by follower neurons in the mushroom body. Earlier, we used a single snapshot in time to represent an odor and found that the PN-KC connectivity had little effect on the Hamming distance between KC representations of the odor. Next, we sought to determine the role of the temporal structure of odor representations in discrimination.

Odor inputs to KCs were modeled as a pattern of spikes from PNs. The statistics of spikes emulated that seen in the extant literature (see Materials and Methods). We simulated trial-trial variability by jittering the spike timing within 50 ms windows. Note that in addition to this jitter, random spikes were inserted such that the mean baseline firing rate in the absence of an odor stimulus was $4 \mathrm{~Hz}$. We simulated different odors by activating different groups of PNs. To visualize the dynamics of the population of PNs, we first calculated the number of spikes generated by each PN in overlapping $50 \mathrm{~ms}$ windows. We then projected the PN activity vector during each $50 \mathrm{~ms}$ window onto the first three principal components. Odor representations of the $\mathrm{PN}$ population may be visualized as continuous trajectories in this reduced-dimensional space. When the odor stimulus was turned on, the AL response followed a trajectory from baseline (defined by low firing rates) to a "fixed point" (Mazor and Laurent, 2005). Once the odor stimulus was turned off, the trajectory returned to baseline, but along a different path from the one it had taken to reach the fixed point after odor onset (Stopfer et al., 2003; Mazor and Laurent, 2005). Multiple trials of the same odor generated trajectories that remained close to each other, while dissimilar odors were well separated in the space defined by the principal components (Fig. 3). The input from PNs was used to drive a population of KCs. In contrast to the threshold model of $\mathrm{KCs}$ used in the previous section, here we modeled KCs as leaky integrate-and-fire neurons with integration properties that matched the responses seen in earlier studies (Perez-Orive et al., 2002, 2004). Here too, we maintained the sparseness of $\mathrm{KC}$ responses across different $\mathrm{PN}-\mathrm{KC}$ connection regimes by choosing progressively higher spike thresholds as the probability of connections increased. The threshold chosen ensured that only $10 \%$ of the KCs spiked in each epoch (50 ms window) when the odor was present regardless of the connectivity. We chose such a threshold-based sparseness to mimic the ultimate effect of the GGN that dynamically adjusts 
feedback inhibition in response to the intensity of the $\mathrm{KC}$ response. However, for high PN-KC connectivity (>50\%), we found that the difference between inputs to different $\mathrm{KCs}$ was very small. Therefore, small changes in the $\mathrm{KC}$ threshold led to an all-or-none response, and consequently to a high variability across trials and a reduced ability to discriminate between odorants. Intrinsic variability in $\mathrm{KC}$ thresholds and differences in the strengths of $\mathrm{PN}-\mathrm{KC}$ synapses can potentially reduce this variability for connectivities $>50 \%$. We used a normalized Hamming distance to visualize differences across all connectivity values. In the $0-50 \%$ connectivity regime, where the number of activated KCs remained nearly the same and well controlled by $\mathrm{KC}$ threshold modification, the Hamming distance matched the normalized Hamming distance except for a constant scaling factor. Including PN temporal patterning revealed some functional differences between different $\mathrm{PN}-\mathrm{KC}$ connectivity regimes.

$\mathrm{KCs}$ received inputs that represented odors with different degrees of similarity between them. We calculated the mean normalized Hamming distance between all pairs of $\mathrm{KC}$ activity vectors for different odors and connectivities (Fig. 4a). Our analysis began to pick out differences in the ability of the KC population with different connectivities to represent odors distinctly. The normalized Hamming distance between $\mathrm{KC}$ odor representations increased with increasing PN-KC connectivity for all odor distances (Fig. 4a). This implied that the representations of two different odors are more distinct in higher connectivity regimes. This could potentially allow the network to accurately associate specific odors with reward signals in downstream layers of the olfactory circuit (Cassenaer and Laurent, 2012; Hige et al., 2015b; Owald et al., 2015). However, an increase in Hamming distance was accompanied by a concomitant increase in the variability of the distance across odor pairs. We found a similar trend in the distance between the trials that represented the same odor (Fig. $4 a$, trace marked $0 \%$ difference). Therefore, for high PN$\mathrm{KC}$ connection densities, it seemed likely that different trials of the same odor could be incorrectly classified as distinct odors. Ideally, the network must maximize the distance between odor representations while also keeping the trial-trial variability within a range that prevents the misclassification of odors. The Hamming distance metric does not take into account the variability of $\mathrm{KC}$ odor representation. Therefore, we used $\mathrm{k}$-medoids clustering to separate the odor representations into nonoverlapping groups. Our data consisted of $25 \mathrm{KC}$ response vectors (5 odors $\times 5$ trials). Each was a 50,000-element-long vector, where each element represented a single $\mathrm{KC}$ and contained either $\mathrm{a} 1$ if that $\mathrm{KC}$ was active or 0 if it was inactive. We determined whether the trials had been grouped correctly based on their odor identity. For each set, we used the percentage of correct classifications as a measure of the ability of the network to distinguish between odorants. As the PN-KC connectivity increased to nearly $45 \%$, the number of correct classifications dropped abruptly, indicating that the distance across trials of the same odor matched or exceeded the distance between representations of different odors (Fig. 4b). Therefore, 45\% PN-KC connectivity increased the distance between representations while keeping trial-trial variability within a reasonable range. This result is similar to that of Jortner (2013), though it is based on the output of KCs over a few seconds of odor stimulation, while Jortner (2013) based the conclusion on a single snapshot of odor input. Next, we used multidimensional scaling to visualize the distribution of different odors on a plane. The algorithm mapped each 50,000 -dimensional KC representations of an odor trial on to a single point on this plane. For low values of $\mathrm{PN}-\mathrm{KC}$ connectivity, multiple trials of the same odor preferentially remained close together. As the divergence of connections increased, the separation between the representations of different trials of a particular odor and different odors began to merge, making it difficult to correctly segregate the odors (Fig. $4 c$, different odors are marked in different colors). The odors plotted here differed from each other in $5 \%$ of the PNs that were stimulated.

It is possible that the differences in Hamming distance could be merely a consequence of using a specific KC model (an integrate-and-fire neuron here) compared with a nonlinear threshold neuron used in earlier sections. To show that this is not the case, we created odor representations in which odors differed only in the identity of PNs that they activated. All active PNs produced the same number of spikes at exactly the same points in time. In this way, we continued to include all aspects of our expanded model but removed any differences in temporal structure that could be used differently by the different connectivity regimes. Therefore, if the usage of our new $\mathrm{KC}$ model that evolved in time was the cause for the functional differences that we saw, then the results of this simulation would differ from that of the previous simulations (Fig. 2c) that used a threshold model. We found that the distance between odor representations in both models, the integrate-and-fire model and the threshold model, were independent of the degree of PN-KC connectivity when temporal features of the odor representation were eliminated (compare Fig. 4d, 2c).

Together, these results suggest that the inclusion of temporal structure in AL activity causes postsynaptic KC populations that receive a large number of inputs to respond differently from those that receive few inputs. However, there appears to be a trade-off here. Dense connectivity regimes are highly sensitive to small changes in incoming input and can incorrectly categorize noisy trials of the same odor as different odors. On the other hand, sparse connectivity regimes produce reliable representations that can be clustered correctly into different groups. However, these are likely to fail if very similar odors are introduced because the representations may not be well separated, as seen from the low Hamming distance between the odor representations

\section{Glomerular organization of the fly aids odor discrimination}

Olfactory receptor neurons in insects are distributed randomly across the antennae within tiny hair-like structures called sensilla. Each receptor neuron expresses a single olfactory receptor protein and possesses a 
a

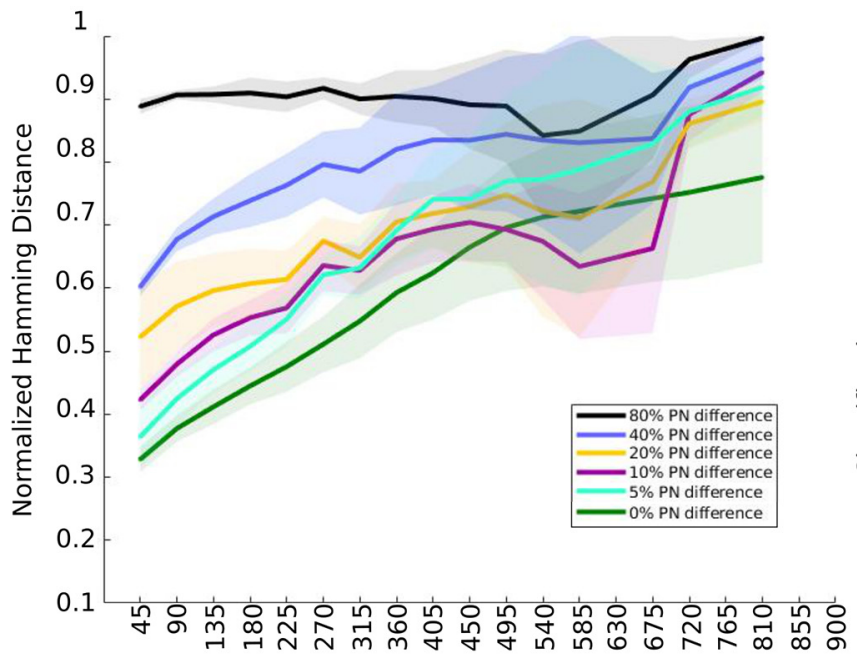

PN-KC Connectvity

C
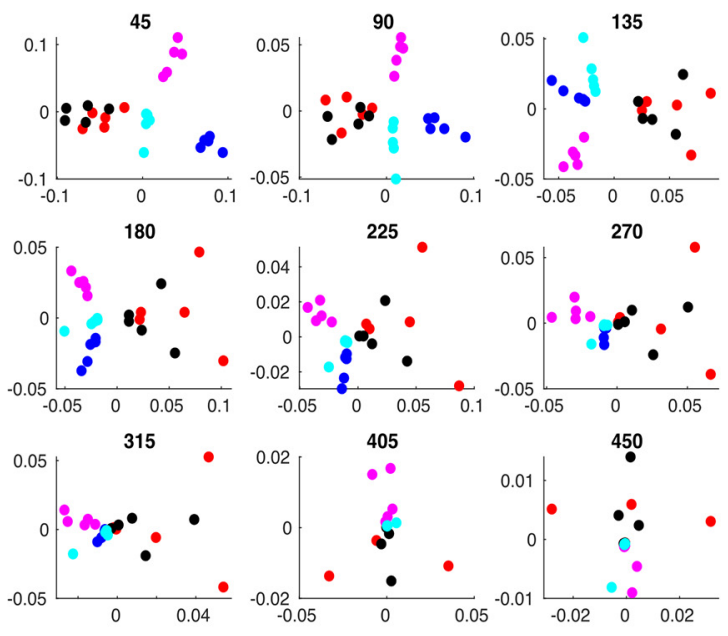

b

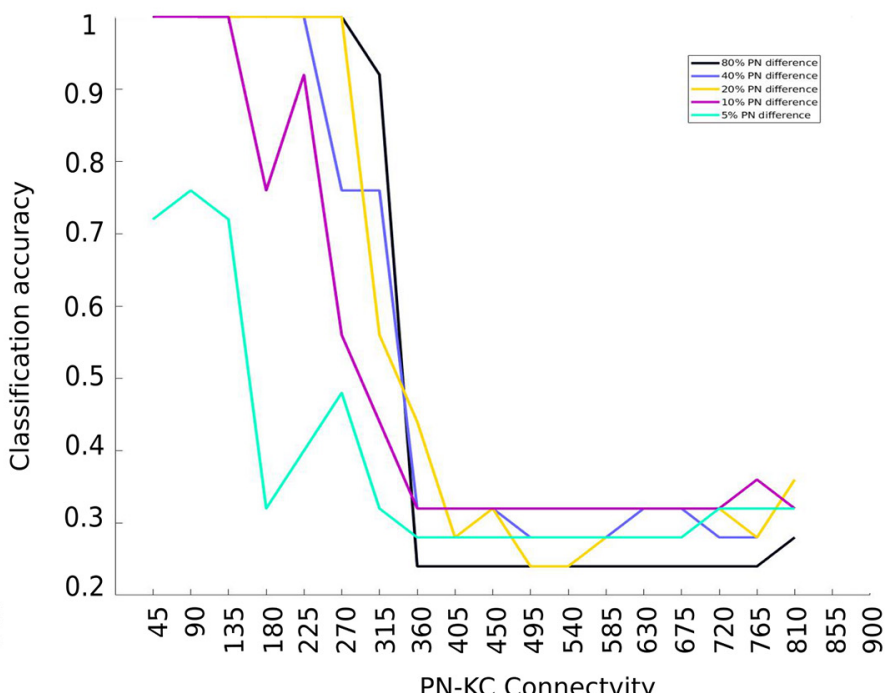

d

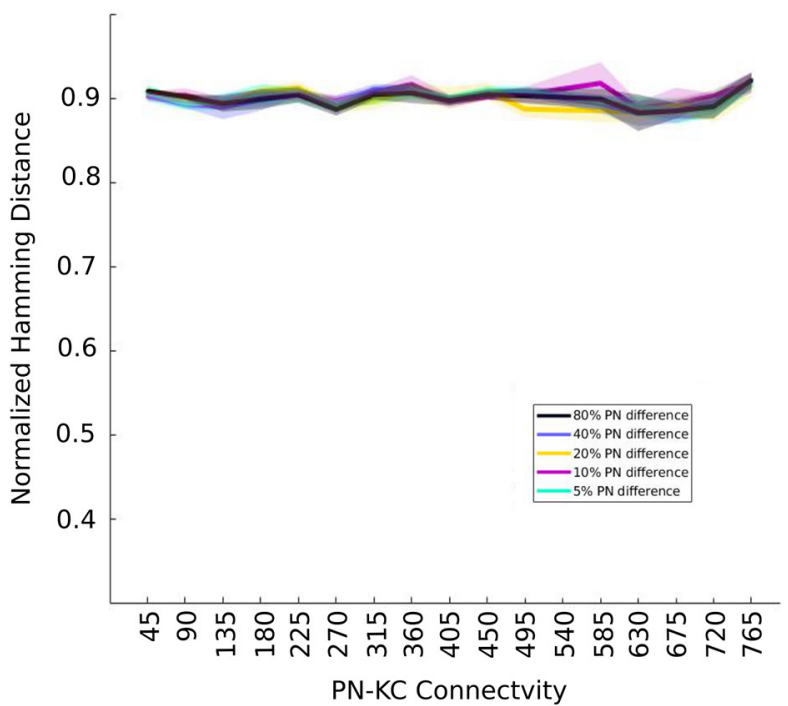

Figure 4. PN temporal patterning reveals the functional differences between connectivities. a, Distance between odor representations. The mean \pm SD normalized Hamming distance between the KC representations of odor pairs is shown as a function of the $\mathrm{PN}-\mathrm{KC}$ connectivity value. Here KCs are modeled as described in Figure $3 . \boldsymbol{b}$, Classification accuracy decreases with increasing $\mathrm{PN}-\mathrm{KC}$ connectivity. A k-medoids clustering algorithm that used the distance between $25 \mathrm{KC}$ activity vectors (five trials $\times$ five odors) was used to categorize each vector as one of five odors. The percentage of correctly classified odor representations is plotted on the $y$-axis as a function of the connectivity of the PN-KC network. c, Odor representations become indistinguishable with increasing PN-KC connectivity. Five odors that differed from each other by $5 \%$ PN input were mapped to a plane using multidimensional scaling. Different trials of a given odor are plotted using a single color. Different odors are plotted using different colors. The PN-KC connectivity is shown in the title of each subplot. $\boldsymbol{d}$, Hamming distance between static odor representations. The mean \pm SD normalized Hamming distance between the KC representations of odor pairs is plotted as a function of PN-KC connectivity. Here, the PN odor representation did not change in time.

receptive field tuned to a variety of odorants (Hallem and Carlson, 2004, 2006). In Drosophila, all the sensory neurons expressing a particular receptor type synapse onto a single glomerulus, giving nearly identical input to sister PNs that receive input from that glomerulus (Kazama and Wilson, 2009). While correlated PN responses can potentially improve the signal-to-noise ratio, this comes at a cost; namely, the dimensionality of the olfactory representation is vastly reduced. The size of the representation may be thought of as the number of independent dimensions; that is, the number of neurons that can generate uncorrelated patterns of activity. In locusts that lack this glomerular organization, the maximum number of independent dimensions is 900 (the number of PNs that could potentially receive unique odor input). In Drosophila, this number reduces dramatically since multiple neurons receive identical input from ORNs and generate a highly correlated output. The number in Drosophila may be 
a

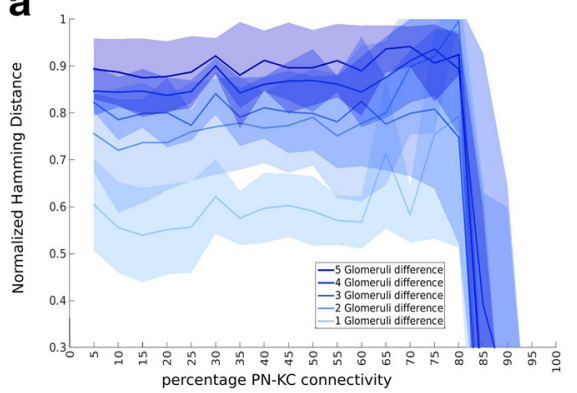

b

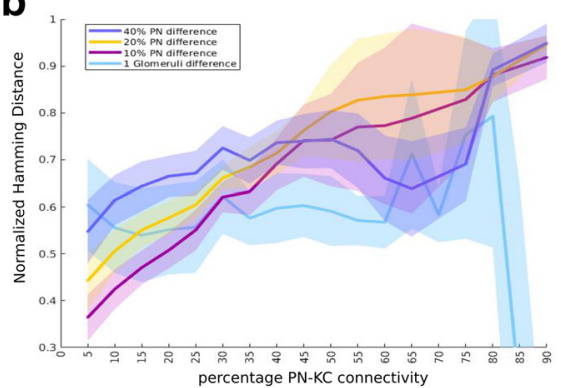

C

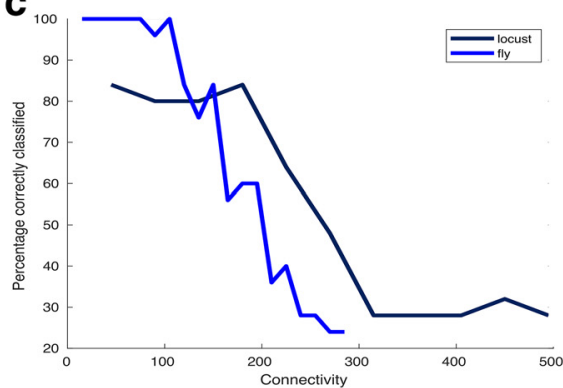

Figure 5. Glomerular organization of the fly aids odor discrimination. $\boldsymbol{a}$, The mean \pm SD normalized Hamming distance as a function of PN-KC connectivity in a network with glomerular structure. $\boldsymbol{b}$, The normalized Hamming distance of odors with a one glomerulus difference in a fly-like glomerular system is compared with the Hamming distance between odor representations of a system with locust-like glomerular structure. c, Classification accuracy of odors that are different by two glomeruli $(2 \%$ or 12 neurons in the fly-like architecture; blue trace) compared with the classification accuracy of odors that differed by $5 \%$ ( 45 neurons) of stimulated odors in locust. Classification accuracy is higher for the fly-like organization for low PN-KC connectivities.

much smaller ( $\sim 50$, the number of glomeruli) since the output of sister PNs is nearly the same. Does the glomerular organization of the Drosophila olfactory system mitigate some of the disadvantages in odor discrimination imposed by sparse $\mathrm{PN}-\mathrm{KC}$ connections?

To test whether the inclusion of the uniglomerular architecture seen in the fly produces any improvement in the ability of sparsely connected networks, we performed simulations in which odors were defined by the glomeruli they activated. These odors differed in the number of unique glomeruli they activated rather than the number of unique PNs (Fig. 5a). These inputs were then fed to the same KC network simulated earlier. We saw that for sparse connectivity regimes the uniglomerular organization magnified the differences in PN activity and increased the Hamming distance between KC representations of odors compared with the nonglomerular case (Fig. 5b). We then used k-medoid-based clustering and classification to determine whether the fly-like architecture provided any benefits in odor classification. We compared the classification accuracy as a function of $\mathrm{PN}-\mathrm{KC}$ connectivity for two cases: a system with a multiglomerular locust-like architecture; and one with a uniglomerular flylike architecture. We found that the uniglomerular architecture improved the classification accuracy of the network for low PN-KC connectivities compared with the multiglomerular architecture (Fig. $5 c$ ). However, this kind of glomerularization appears to cause no change or even to slightly reduce the ability of dense connectivity schemes to separate odor representations. This suggests that the glomerular organization seen in the fly does in fact improve the ability of the animal to distinguish between odors.

\section{Discussion}

\section{Discrimination of purely spatial odor representations is independent of PN-KC connection density}

In the locust AL, PNs generate elaborate spatiotemporal patterns in response to an odor. These patterns are read by KCs in the MB. The density of connections between PNs and $\mathrm{KCs}$ is such that each $\mathrm{KC}$ receives input from nearly one-half of the PNs. A $50 \%$ probability of connections from PNs to KCs ensures that the PN inputs to $\mathrm{KCs}$ are maximally separated. The number of ways to pick $m$ of $n$ elements is maximized when $m=\frac{n}{2}$, thus maximizing the distance between inputs to KCs (Fig. 2b; Jortner, 2013). This argument assumed that this distance between inputs dropped off quickly as $m$ changed from $m=\frac{n}{2}$. Therefore, in schemes that did not have close to $50 \%$ connectivity, KCs did not receive sufficiently distinct inputs. We found that while the inputs were indeed maximally separated at $50 \%$ connectivity, once the summed inputs underwent a KC threshold function, all connectivity regimes were equally good at separating odors. This is in line with more recent studies that show that even a $5 \%$ connection probability generates a large representation space such that even highly similar odors are mapped to distant locations (Litwin-Kumar et al., 2017). However, these observations are confined to odor representations that are static. When the temporal patterning of inputs was included, denser connectivities appeared to be significantly better at separating odor representations.

\section{Odor representations are variable in networks with dense connectivity}

Increasing connection density comes at a price. Odorants are embedded in a noisy and changing milieu. Recognition of appetitive and aversive odorants must play out against a background of irrelevant olfactory information. Thus, the network must be tolerant to perturbations in the odor representation. This constraint introduces an upper bound on the density of connections between PNs and KCs. Our simulations demonstrated that high connectivity values led to highly variable representations of the odor by KCs, as was seen from the SD of the Hamming distance. Dense (80-95\%) connectivity regimes generated representations that were four to five times more variable than representations generated by sparse connectivity schemes. The reason for this increased variability is that for dense connectivity schemes, KCs see nearly identical input from PNs. For connectivity regimes $>50 \%$, with 
temporally varying $\mathrm{PN}$ inputs, the discriminability between KC inputs decreases with increasing connection density. The response of KCs is modulated by inhibitory feedback from the GGN. The GGN inhibits all of the KCs and maintains sparseness across large variations in odor attributes by controlling the propensity of KCs to respond. In highconnectivity regimes, a threshold that causes one of the $\mathrm{KCs}$ to fire invariably allows most KCs to fire. A small increase in threshold can lead to a condition where none of the KCs fire. Noisy changes in input statistics can thus drive the $K C$ responses leading to large trial-trial variability. While the variability of the odor representation is maximal for connection densities in the $80-95 \%$ range, as mentioned previously, even networks with connection densities in the range of $45-60 \%$ show poor classification ability when exposed to multiple trials of the same odor. This is clearly not ideal for a system attempting to represent sensory information in a stereotyped way over different trials and learn from experience.

\section{Temporal patterning of $\mathrm{PN}$ activity reveals functional differences among $\mathrm{PN}-\mathrm{KC}$ connectivity regimes}

A key insight from the simulations performed in this study is the observation that the categorization of odors in the insect $\mathrm{MB}$ is dependent on an interaction between $\mathrm{PN}-\mathrm{KC}$ connectivity and temporal patterning of PN input. The reason for these differences, as shown earlier, is due to the differing demands of connectivity regimes on the temporal coincidence of spiking and spike thresholds. Together, our results reiterate that temporal patterning of PN input carries information about the identity of odors (Stopfer et al., 2003). But, more importantly, we show that this information can be used differently by systems with different PN-KC connectivity values. Sparse connectivity regimes use this in a way that allows for reduction in noise sensitivity, and dense connectivity regimes use it to maximally separate between odors. Given the complexity of our sensory world, the olfactory system must balance two seemingly conflicting goals: resolve highly similar sensory inputs and do so with considerable reliability despite noisy variations in the input. Our model suggests that the locust and Drosophila live in different regimes of a continuum of possibilities, arriving at different solutions, perhaps driven by their own evolutionary histories. Importantly, the differences in the functions of these two circuits are only revealed when the temporal structure of the odor representation is taken into account.

\section{References}

Albus JS (1971) A theory of cerebellar function. Math Biosci 10:2561.

Aso $Y$, Sitaraman D, Ichinose $T$, Kaun KR, Vogt $K$, Belliart-Guérin G, Plaçais P-Y, Robie AA, Yamagata N, Schnaitmann C, Rowell WJ, Johnston RM, Ngo T-TB, Chen N, Korff W, Nitabach MN, Heberlein U, Preat T, Branson KM, Tanimoto H, et al (2014) Mushroom body output neurons encode valence and guide memory-based action selection in Drosophila. Elife 3:e04580.

Bazhenov M, Stopfer M, Rabinovich M, Abarbanel HDI, Sejnowski TJ, Laurent G (2001) Model of cellular and network mechanisms for odor-evoked temporal patterning in the locust antennal lobe. Neuron 30:569-581.

Caron SJC, Ruta V, Abbott LF, Axel R (2013) Random convergence of olfactory inputs in the Drosophila mushroom body. Nature 497:113-117.

Cassenaer S, Laurent G (2012) Conditional modulation of spike-timing-dependent plasticity for olfactory learning. Nature 487:128128.

Destexhe A, Mainen ZF, Sejnowski TJ (1994) An efficient method for computing synaptic conductances based on a kinetic model of receptor binding. Neural Comput 6:14-18.

Dhawale AK, Hagiwara A, Bhalla US, Murthy VN, Albeanu DF (2010) Non-redundant odor coding by sister mitral cells revealed by light addressable glomeruli in the mouse. Nat Neurosci 13:1404-1412.

Fisek M (2014) Connectivity and computations in higher-order olfactory neurons in Drosophila. PhD thesis, Harvard University.

Gruntman E, Turner GC (2013) Integration of the olfactory code across dendritic claws of single mushroom body neurons. Nat Neurosci 16:1821-1829.

Hallem EA, Carlson JR (2004) The odor coding system of Drosophila. Trends Genet 20:453-459.

Hallem EA, Carlson JR (2006) Coding of odors by a receptor repertoire. Cell 125:143-160.

Heisenberg M (2003) Mushroom body memoir: from maps to models. Nat Rev Neurosci 4:266-275.

Hige T, Aso Y, Modi MN, Rubin GM, Turner GC (2015a) Heterosynaptic plasticity underlies aversive olfactory learning in Drosophila. Neuron 88:985-998.

Hige T, Aso Y, Rubin GM, Turner GC (2015b) Plasticity-driven individualization of olfactory coding in mushroom body output neurons. Nature 526:258-262.

Hopfield JJ, Tank DW (1986) Computing with neural circuits: a model. Science 233:625-633.

Jortner RA (2013) Network architecture underlying maximal separation of neuronal representations. Front Neuroeng 5:19.

Jortner RA, Farivar SS, Laurent G (2007) A simple connectivity scheme for sparse coding in an olfactory system. J Neurosci 27:1659-1669.

Kazama H, Wilson RI (2009) Origins of correlated activity in an olfactory circuit. Nat Neurosci 12:1136-1144.

Kristan WB, Katz P (2006) Form and function in systems neuroscience. Curr Biol 16:R828-31.

Laurent G (1996) Dynamical representation of odors by oscillating and evolving neural assemblies. Trends Neurosci 19:489-496.

Laurent G, Wehr M, Davidowitz H (1996) Temporal representations of odors in an olfactory. J Neurosci 16:3837-3847.

Lin AC, Bygrave AM, de Calignon A, Lee T, Miesenböck G (2014) Sparse, decorrelated odor coding in the mushroom body enhances learned odor discrimination. Nat Neurosci 17:559-568.

Litwin-Kumar A, Harris KD, Axel R, Sompolinsky H, Abbott LF (2017) Optimal degrees of synaptic connectivity. Neuron 93:1153-1164.

Marr D (1969) A theory of cerebellar cortex. J Physiol 202:437-470.

Masuda-Nakagawa LM, Ito K, Awasaki T, O'Kane CJ (2014) A Single GABAergic neuron mediates feedback of odor-evoked signals in the mushroom body of larval Drosophila. Front Neural Circuits 8:35.

Mazor O, Laurent G (2005) Transient dynamics vs fixed points in odor representations by locust antennal lobe projection neurons. Neuron 48:661-673.

Owald D, Felsenberg J, Talbot CB, Das G, Perisse E, Huetteroth W, Waddell S (2015) Activity of defined mushroom body output neurons underlies learned olfactory behavior in Drosophila. Neuron 86:417-427.

Papadopoulou M, Cassenaer S, Nowotny T, Laurent G (2011) Normalization for sparse encoding of odors by a wide-field interneuron. Science 332:721-725. 
Perez-Orive J, Mazor O, Turner GC, Cassenaer S, Wilson RI, Laurent G (2002) Oscillations and sparsening of odor representations in the mushroom body. Science 297:359-366.

Perez-Orive J, Bazhenov M, Laurent G (2004) Intrinsic and circuit properties favor coincidence detection for decoding oscillatory input. J Neurosci 24:6037-6047.

Saha D, Sun W, Li C, Nizampatnam S, Padovano W, Chen Z, Chen A, Altan E, Lo R, Barbour DL, Raman B (2017) Engaging and disengaging recurrent inhibition coincides with sensing and unsensing of a sensory stimulus. Nat Commun 8:15413.

Stopfer M, Jayaraman V, Laurent G (2003) Intensity vs identity coding in an olfactory system. Neuron 39:991-1004.

Tanaka NK, Ito K, Stopfer M (2009) Odor-evoked neural oscillations in Drosophila are mediated by widely branching interneurons. J Neurosci 29:8595-8603.
Turner GC, Bazhenov M, Laurent G (2008) Olfactory representations by Drosophila mushroom body neurons. J Neurophysiol 99:734746.

Wehr M, Laurent G (1996) Odour encoding by temporal sequences of firing in oscillating neural assemblies. Nature 384:162-166.

Wiechert MT, Judkewitz B, Riecke H, Friedrich RW (2010) Mechanisms of Pattern Decorrelation by Recurrent Neuronal Circuits. Nat Neurosci 13:1003-1010.

Wilson RI, Laurent G (2005) Role of GABAergic inhibition in shaping odor-evoked spatiotemporal patterns in the Drosophila antennal lobe. J Neurosci 25:9069-9079.

Yasuyama K, Salvaterra PM (1999) Localization of choline acetyltransferase-expressing neurons in Drosophila nervous system. Microsc Res Tech 45:65-79. 